\title{
Cisto Ósseo Traumático - Relato de caso
}

\author{
Traumatic Bone Cyst - Case report \\ Quiste Óseo Traumático - Reporte de caso
}

\author{
Híttalo Carlos Rodrigues de Almeida \\ ORCID: https://orcid.org/0000-0001-7348-6601 \\ Universidade de Pernambuco, Brasil \\ E-mail: hittalo.rodrigues@ hotmail.com \\ Zilda Betânia Barbosa Medeiros de Farias \\ ORCID: https://orcid.org/0000-0001-9549-2260 \\ Universidade de Pernambuco, Brasil \\ E-mail: zfarias2011@ @otmail.com \\ Bruna Peixoto Nogueira dos Santos \\ ORCID: https://orcid.org/0000-0003-4068-5887 \\ Universidade de Pernambuco, Brasil \\ E-mail: brunanogueira_cd@ @otmail.com \\ Rebeka Thiara Nascimento dos Santos \\ ORCID: https://orcid.org/0000-0002-0422-8593 \\ Universidade de Pernambuco, Brasil \\ E-mail: rebeka.nsantos@gmail.com \\ Luiz Pedro Mendes de Azevedo \\ ORCID https://orcid.org/0000-0003-0132-5539 \\ Universidade de Pernambuco, Brasil \\ E-mail: luiz.pedro@upe.br \\ Pâmella Recco Álvares \\ ORCID: https://orcid.org/0000-0003-3396-9339 \\ Universidade de Pernambuco, Brasil \\ E-mail: pamellarecco@ hotmail.com \\ Rômulo Oliveira de Hollanda Valente \\ ORCID: https://orcid.org/0000-0002-2806-9945 \\ Hospital Getúlio Vargas, Brasil \\ E-mail: mcristinavo@hotmail.com \\ Márcia Maria Fonseca da Silveira \\ ORCID: https://orcid.org/0000-0002-2611-5661 \\ Universidade de Pernambuco, Brasil \\ E-mail: marcia.silveira@upe.br \\ Ana Paula Veras Sobral \\ ORCID: https://orcid.org/0000-0002-0801-9385 \\ Universidade de Pernambuco, Brasil \\ E-mail: ana.sobral@upe.br
}

\begin{abstract}
Resumo
O cisto ósseo traumático (COT) é uma lesão não-neoplásica delimitada por uma fina camada de tecido conjuntivo frouxo, sem revestimento epitelial. Sua etiologia e patogênese são incertas, sendo o trauma um dos possíveis fatores no surgimento desse cisto intraósseo. Os locais mais acometidos são ossos longos e gnáticos. Nos ossos gnáticos, surgem com maior frequência na região posterior e anterior da mandíbula, geralmente são assintomáticos e descobertos em exames radiográficos de rotina. O diagnóstico definitivo de cisto ósseo traumático é obtido através da análise das características clínicas e radiográficas da lesão, bem como durante o procedimento de biópsia e análise histopatológica. O objetivo desse trabalho é relatar um caso clínico de cisto ósseo traumático com acompanhamento pós-cirúrgico.
\end{abstract}

Palavras-chave: Cistos ósseos; Mandíbula; Cistos não odontogênicos.

\begin{abstract}
Traumatic bone cyst (TOC) is a non-neoplastic lesion delimited by a thin layer of loose connective tissue, without an epithelial lining. Its etiology and pathogenesis are uncertain, and trauma is one of the possible factors in the emergence of this intraosseous cyst. The most affected sites are long and gnathic bones. In the gnathic bones, they appear more frequently in the posterior and anterior region of the mandible, they are generally asymptomatic and discovered in routine radiographic exams. The definitive diagnosis of traumatic bone cyst is obtained by analyzing the clinical and radiographic characteristics of the lesion, as well as during the biopsy procedure and histopathological analysis. The objective of this work is to report a clinical case of traumatic bone cyst with post-surgical follow-up.
\end{abstract}

Keywords: Bone cysts; Jaw; Non-odontogenic cysts. 


\section{Resumen}

El quiste óseo traumático (COT) es una lesión no neoplásica delimitada por una fina capa de tejido conectivo laxo, sin revestimiento epitelial. Su etiología y patogenia son inciertas y el trauma es uno de los posibles factores en la aparición de este quiste intraóseo. Los sitios más afectados son los huesos largos y gnáticos. En los huesos gnáticos, aparecen con mayor frecuencia en la región posterior y anterior de la mandíbula, generalmente son asintomáticos y se descubren en los exámenes radiográficos de rutina. El diagnóstico definitivo de quiste óseo traumático se obtiene analizando las características clínicas y radiográficas de la lesión, así como durante el procedimiento de biopsia y análisis histopatológico. El objetivo de este trabajo es reportar un caso clínico de quiste óseo traumático con seguimiento posquirúrgico.

Palabras clave: Quistes óseos; Mandíbula; Quistes no odontogénicos.

\section{Introdução}

O cisto ósseo traumático (COT) é uma lesão intraóssea benigna caracterizada por uma cavidade vazia ou com conteúdo líquido seroso ou sero-sanguinolento delimitada por uma fina camada de tecido conjuntivo frouxo sem revestimento epitelial (Rocha et al., 2013). Em 90\% dos casos está localizada nos ossos longos, seguido de 10\% ossos gnáticos (Mannarino et al., 2014). Representa aproximadamente $1 \%$ de todos os cistos maxilares, acometendo as regiões de corpo e sínfise de mandíbula com maior frequência (Velho, 2015; Kumar et al. 2011).

Este cisto ocorre em indivíduos jovens, principalmente durante a segunda década de vida (Harnet et al., 2008). Sua etiologia e patogênese são incertas, porém na literatura são levantadas hipóteses e a teoria mais aceita é a de origem traumática, que levaria à hemorragia intra-óssea e consequente liquefação do coágulo, levando ao desenvolvimento do cisto (Valladares et al., 2008). É uma lesão que possui a característica peculiar de geralmente não apresentar expansão da cortical e/ou reabsorção radicular (Velho, 2015), com respostas positivas aos testes de sensibilidade pulpar dos dentes envolvidos na área da lesão.

Clinicamente, a lesão apresenta-se assintomática, embora alguns pacientes relatem sensibilidade dentária ou parestesia (Andrade et al., 2016). Frequentemente é diagnosticada em exames radiográficos de rotina, apresentando uma imagem radiolúcida unilocular, bem delimitada e com margens festonadas. As características radiográficas do cisto ósseo traumático não são suficientes para o diagnóstico definitivo, pois se assemelha a outras lesões dos maxilares (Mannarino et al., 2014). A exploração cirúrgica é necessária para auxiliar no diagnóstico e o material obtido na curetagem da cavidade deve ser encaminhado para exame anatomopatológico (Andrade et al., 2016; Kumar et al., 2011).

Este artigo tem por objetivo relatar um caso de cisto ósseo traumático em mandíbula abordando suas características clínicas, radiográficas e proservação.

\section{Metodologia}

O presente estudo corresponde a um relato de caso, caracterizando-se como um estudo descritivo, utilizandose de imagens para seu auxílio e ilustração, sendo conduzido da forma mais tradicional de estratégia de pesquisa, apoiado nas técnicas de observação direta e revisão de literatura, integrando-se de um método abrangente de coleta e análise de dados, compreendendo uma completa e clara descrição e discussão de fatores que vão desde o diagnóstico até o tratamento da condição (Pereira et al., 2018). Obedecendo as questões éticas que envolvem este tipo de pesquisa, de forma atinente à resolução nº46/2012 aprovada pelo Conselho Nacional de Saúde, que rege as pesquisas e testes em seres humanos. Este estudo foi aprovado pela comissão de ética da Universidade de Pernambuco (UPE) de acordo com a declaração de Helsinque, a responsável legal pelo menor assinou o termo de consentimento livre e esclarecido (TCLE) para participação na pesquisa, autorizando a utilização de dados de seu caso clínico/cirúrgico e documentação fotográfica. 


\section{Relato de Caso}

Paciente 16 anos de idade, sexo masculino foi encaminhado ao Estomatologista devido à lesão radiolúcida multilocular localizada na região posterior da mandíbula do lado esquerdo, em direção ao ramo ascendente, identificada em exame radiográfico de rotina (Figura 1). Não foram observados sinais de reabsorção radicular e/ou deslocamento de dentes. Ao exame físico, foi observado expansão da cortical vestibular da mandíbula e discreta assimetria facial. A percussão e aos testes de sensibilidade pulpar demonstraram vitalidade dos dentes próximos à lesão.

Figura 1 - Lesão multilocular localizada na região posterior de mandíbula do lado esquerdo envolvendo o dente 38 parcialmente irrompido.

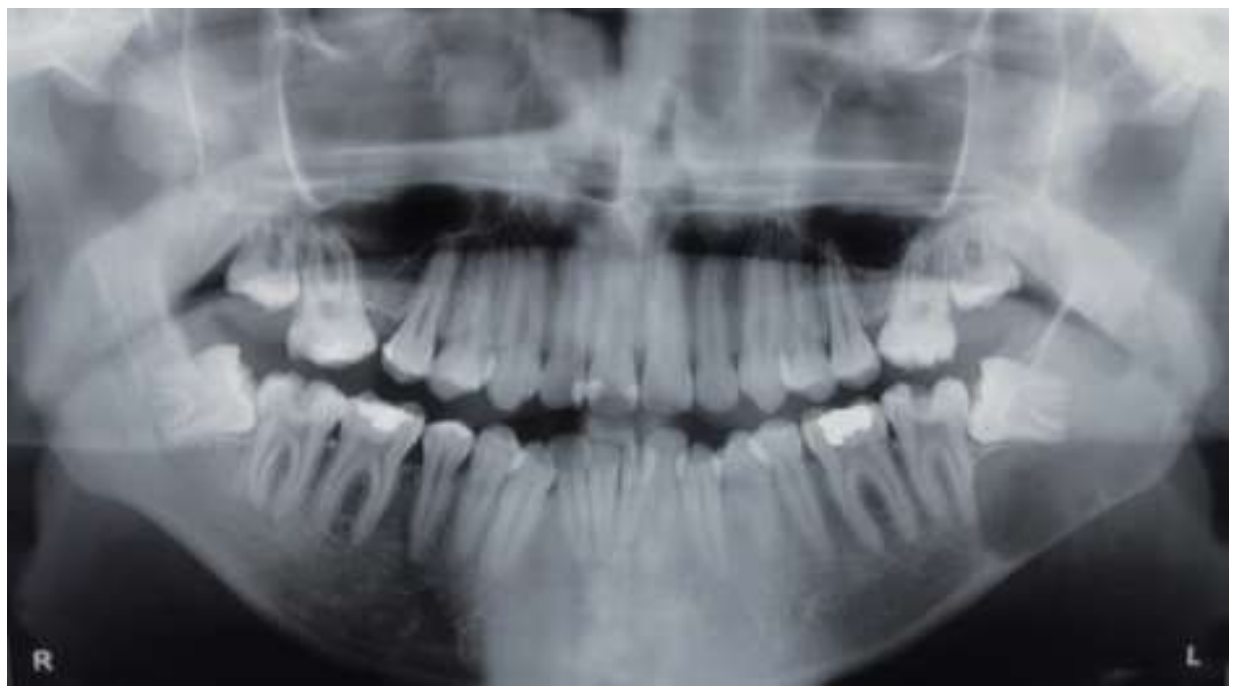

Fonte: Autores.

Foi realizado punção aspirativa antes da exploração cirúrgica, e o conteúdo foi de líquido sanguinolento (Figura 2). Posteriormente, exploração cirúrgica com acesso à região da lesão, osteotomia para remoção de fragmento, o qual foi enviado ao laboratório de patologia para análise anatomopatológica, sendo removido o dente 38.

Figura 2 - Punção aspirativa com conteúdo líquido sanguinolento.

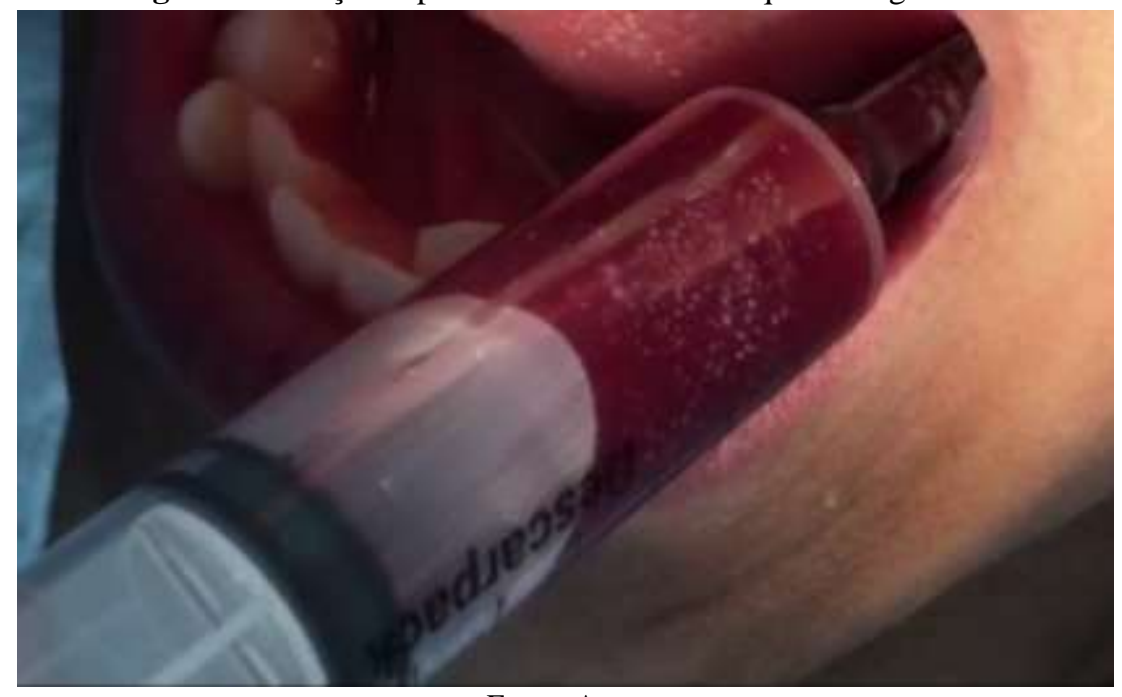

Fonte: Autores. 
Os cortes histológicos revelaram fragmentos de mucosa revestidos por epitélio pavimentoso estratificado. A lâmina própria apresentou um moderado infiltrado inflamatório monocelular; na porção de trabécula óssea foi observada área fibromixomatosas espessadas entre as trabéculas (Figura 3), cujo diagnóstico foi de cisto ósseo traumático.

Figura 3 - Presença de infiltrado inflamatório monocelular e áreas fibromixomatosas espessadas: A. (100XH.E) B. Detalhe da figura A (400X H.E).
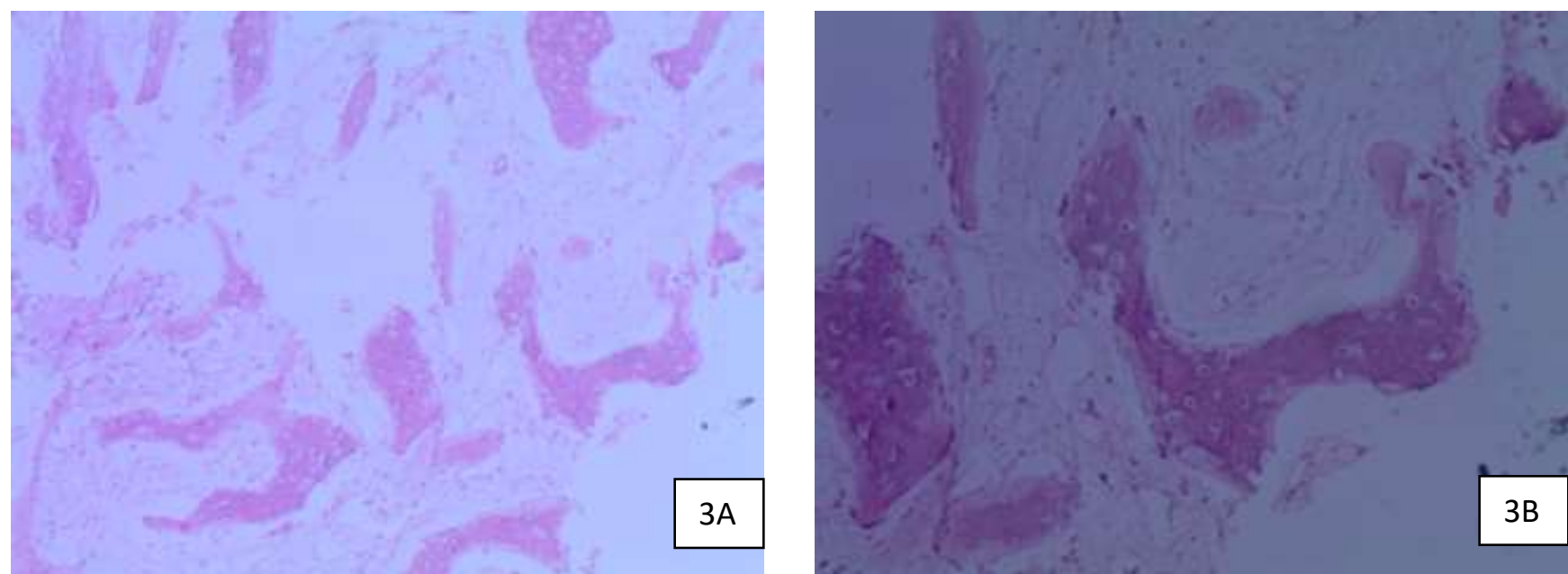

Fonte: Autores.

O acompanhamento radiográfico foi realizado com três e seis meses com radiografias panorâmicas sendo observado neoformação óssea. (Figura 4).

Figura 4 - Área da lesão: A. Imagem inicial da lesão. B. Controle de três meses. C. Controle de seis meses.
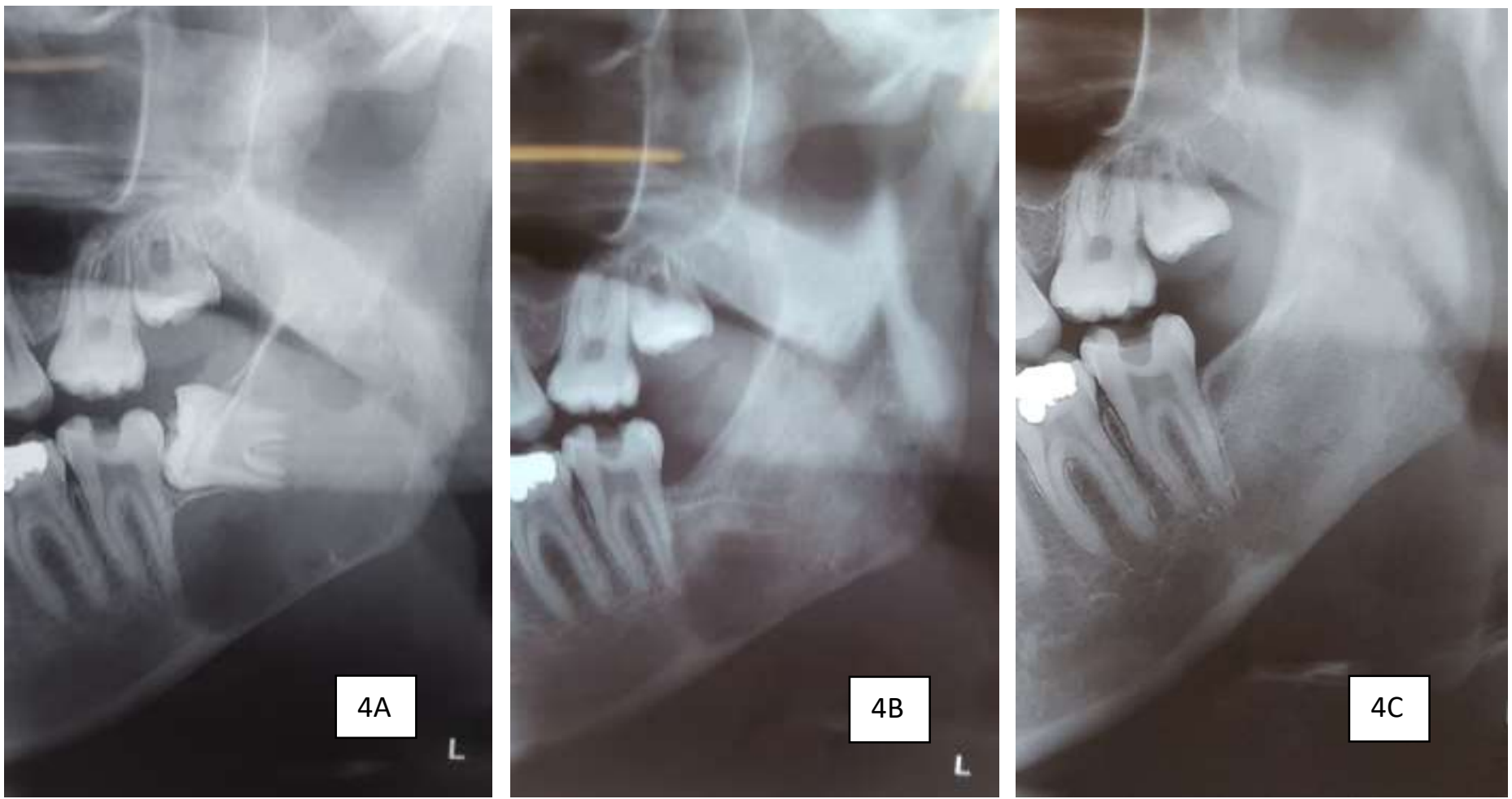

Fonte: Autores. 


\section{Discussão}

Cistos ósseos traumáticos são comumente encontrados em ossos longos e coluna vertebral, representando $2 \%$ dos cistos relatados na face (Andrade et al., 2016). Os casos observados na mandíbula possuem maior incidência em regiões posteriores (corpo e ramo). Embora tenha sido descrito no início do século XX, a patogênese do COT permanece incerta e sujeita a controvérsias (Martins-Filho et al., 2012).

A mandíbula por ser um osso mais cortical, a teoria trauma-hemorragia é a mais aceita e pode estar relacionada à prevalência da lesão, devido à menor capacidade de reparação deste osso quando comparado à maxila (Velho, 2015). Entretanto, os relatos de trauma na região de desenvolvimento da lesão são infrequentes, como observado no presente estudo, com a possibilidade de que microtraumas nos dentes e no rebordo alveolar participem da patogênese do COT (Kumar et al., 2011; Martins-Filho et al., 2012; Velho, 2015). No caso clínico relatado, a localização da lesão envolveu o ramo mandibular, semelhante ao caso apresentado por Mannarino et al., 2014.

Os COT são geralmente diagnosticados em pacientes jovens, com idades inferiores a 20 anos de idade, sendo a maior incidência reportada no sexo masculino (Andrade et al., 2016; et al., 2011; Rocha et al., 2013), em concordância com o caso relatado. Radiograficamente, apresenta-se como uma área radiolúcida unilocular na região posterior da mandíbula, com as margens festonadas entre as raízes dentárias, podendo ter associação com dentes inclusos (Andrade et al., 2016; Mannarino et al., 2014; Martins-Filho et al., 2012; Rocha et al., 2013).

Entretanto, neste caso, a aparência radiográfica da lesão foi sugestiva de outras patologias dos maxilares, como ameloblastoma e queratocístico odontogênico devido a sua aparência multilocular. Segundo o estudo apresentado por Martins Filho et. al 2012 é comum variações neste padrão radiográfico, como a presença de multiloculações, associação com dentes inclusos/impactados e múltiplas ocorrências em um mesmo paciente.

Diversas lesões intraósseas radiolúcidas podem acometer os maxilares, sendo a maioria delas assintomáticas e descobertas em exames radiográficos de rotina (Jesus, 2010; Tong; NG; Yan, 2003). O diagnóstico diferencial das lesões radiolúcidas dos maxilares, inclui: cisto periodontal lateral, granuloma central de células gigantes, fibroma ameloblástico, cisto dentígero, queratocístico odontogênico, tumor odontogênico adenomatóide e ameloblastoma (Mannarino et al., 2014).

O exame radiográfico não é suficiente para fechar o diagnóstico, que é sugestivo durante a cirurgia, quando uma cavidade óssea vazia, sem revestimento epitelial, que pode conter líquido, é encontrada (Harnet et al., 2008; Martins-Filho et al., 2012). A abertura da cavidade promove depósito de sangue, seguido de organização do coágulo e neoformação óssea (Andrade et al., 2016).

Histologicamente, o COT revela a presença de uma membrana de tecido conjuntivo recobrindo as paredes da cavidade patológica. Cristais de colesterol, focos hemorrágicos e osteoclastos podem estar presentes (Paiva et al., 2011; Lago et al., 2006; Martins-Filho et al., 2012) Contudo, o diagnóstico definitivo de COT é quase invariavelmente realizado no momento da exploração cirúrgica da lesão, uma vez que o material coletado para análise histológica na maioria das vezes é escasso, em decorrência da dificuldade na remoção da fina membrana conjuntiva.

A neoformação óssea é observada dentro de poucos meses pós-operatório e apresenta, geralmente, remissão da lesão com contornos ósseos preservados, sem reabsorção radicular (Andrade et al., 2016; Oliveira et al., 2012; Rocha et al., 2013; Tong; Ng; Yan, 2003). A curetagem é considerada o tratamento de escolha e a recidiva é incomum (Martins-Filho et al., 2012; Valladares et al., 2008). O acompanhamento radiográfico periódico deve ser realizado até a remissão da lesão. 


\section{Considerações Finais}

Como as características desse cisto podem ser variadas, o exame clínico cuidadoso e a avaliação radiográfica juntamente com os sinais e sintomas clínicos têm um papel muito significativo no diagnóstico definitivo, tratamento apropriado e avaliação precisa do prognóstico.

\section{Conflitos de interesse}

Os autores não têm conflito de interesses a declarar.

\section{Referências}

Aggarwal, V.; \& Singla, M. (2010) Use of computed tomography scans and ultrasound in differential diagnosis and evaluation of non-surgical management of periapical lesions. Endodontology. 109(6):917-23.

Andrade, E. L.; Cerqueira, L. S.; Rebouças, D. S; Ferreira, T. G.; \& Marchionni, A. M. T. (2016) Cisto ósseo simples: relato de caso. Revista de Cirurgia e Traumatologia Buco-maxilo-facial; 16(2): 36-39.

Bava, F. A., Umar, D., Bahseer, B., \& Baroudi, K. (2015). Bilateral radicular cyst in mandible: an unusual case report. Journal of International Oral Health.. $7(2) 61-63$.

Corrêa, M.; Elias, R.; Cherubim, K.; \& Ponzoni, D. (2002). Cisto Radicular Residual: Relato de Caso Clínico. Jornal Brasileiro de Clínica Odontológica Integrada. 6(32):133-35.

Garcia, J. C. M., Gaetti, E. C. J., Santos, C. M., Masocatto, D. C., Quadros, D. C., Oliveira, M. M., Macena, J. Á., \& Teixeira, F. R. (2015) Cisto periapical residual: relato de caso clínico-cirúrgico. Arch Health Invest 4(1): 45-49.

Graziani, M. (1995) Cirurgia buco maxilo facial. Ed. Guanabara/Koogan.

Harnet, J. C., Lombardi, T., Klewansky, P., Rieger, J., Tempe, M. H., \& Clavert, J. M. (2008) Solitary bone cyst of the jaws: a review of the etiopathogenic hypotheses. J Oral Maxillofac Surg; 66(11):2345-2348.

Kumar, N. D., Sherubin, J. E., Raman, U., \& Shettar, S. (2011). Solitary bone cyst. Indian J Dent Res ;22(1):172-174.

Mannarino, F. S.; Gorla, L. F. O.; Gabrielli, M. F. R.; \& Vieira, E. H. (2014) Cisto ósseo simples - relato de casos. Revista de Cirurgia e Traumatologia Bucomaxilo-facial. 14(2):15-20

Martins-Filho, P.R., Santos, T., Araújo, V. L., Santos, J.S., Andrade, E. S., \& Silva, L. C. (2012). Traumatic bone cyst of the mandible: a review of 26 cases. Braz J Otorhinolaryngol;78(2):16-21.

Nobuhara, W. \& Del Rio, C. (1993). Incidence of periradicular panthoses in endodontic treatment failures. J Endod. 19(6):315-318.

Oliveira, D. H. I. P., Lima, E. N. A., Araújo, C. R. F., Germano, A. R., Medeiros, A. M. C., \& Queiroz, L. M. G. (2011). Cisto residual com grande dimensão: relato de caso e revisão da literatura. Revista de Cirurgia e Traumatologia Buco-maxilo-facial; 11(2): 21-26.

Peker E., Ogutlu F., Karaca I. R., Gultekin E. S., \& Cakir M. (2016). 5 year retrospective study of biopsied jaw lesions with the assessment of concordance between clinical and histopathological diagnoses. Journal of Oral and Maxillofacial Pathology.20(1)78-85.

Pereira J. F., Milagres R. M., Andrade B. A. B., Messora M. R., \& Kawata L. T. (2012). Extensive Radicular Cyst: a Case Report. Revista de Cirurgia e Traumatologia Buco-maxilo-facial. 12(2):37-42.

Valladares, C. P., Israel, M. S., Noleto, J. W., Braga, C. L. S., Lourenço, S. Q. C., \& Dias, E..P. (2008). isto ósseo simples em pacientes sob tratamento ortodôntico: relato de dois casos. R Dental Press Ortodon Ortop Facial. 13(2): 132-137.

Velho, F. M. T. (2015) Cisto Ósseo Traumático na Região da Sínfise Mandibular: Relato de caso. Monografia [Especialização em Radiologia e Imaginologia]. Porto Alegre: Universidade Federal do Rio Grande do Sul. 